\title{
Evidence of a founder effect for the tissue-nonspecific alkaline phosphatase (TNSALP) gene E174K mutation in hypophosphatasia patients
}

\author{
Muriel Hérasse ${ }^{1}$, Marc Spentchian ${ }^{2}$, Agnès Taillandier ${ }^{3}$ and Etienne Mornet $^{\star, 1,3}$ \\ ${ }^{1}$ Laboratoire de Cytogénétique et Génétique Moléculaire Humaine, Université de Versailles-Saint Quentin en Yvelines, \\ Versailles, France; ${ }^{2}$ Service de Biochimie, Centre Hospitalier de Versailles, Versailles, France; ${ }^{3}$ Laboratoire SESEP, \\ Université de Versailles-Saint Quentin en Yvelines, Versailles, France
}

Hypophosphatasia is a rare inborn error of metabolism characterised by defective bone mineralisation caused by a deficiency of liver-, bone- or kidney-type alkaline phosphatase due to mutations in the tissuenonspecific alkaline phosphatase (TNSALP) gene. The clinical expression of the disease is highly variable, ranging from stillbirth with a poorly mineralised skeleton to pathologic skeletal fractures which develop in late adulthood only. This clinical heterogeneity is due to the strong allelic heterogeneity in the TNSALP gene. We found that mutation E174K is the most frequent in Caucasian patients, and that it was carried by $31 \%$ of our patients with mild hypophosphatasia. Because the mutation was found in patients of various geographic origins, we investigated whether it had a unique origin or rather multiple origins due to recurrence of de novo mutations. Three intragenic polymorphisms, S93S, 472+12delG and V505A, were genotyped in patients carrying E174K and in normal unrelated individuals. Our results show that all the E174K mutations are carried by a common ancestral haplotype, also found at low frequency in normal and hypophosphatasia chromosomes. We conclude that the TNSALP gene E174K mutation is the result of a relatively ancient ancestral mutation that occurred on a single chromosome in the north of Western Europe and spread throughout the rest of Europe and into the New World as a result of human migration.

European Journal of Human Genetics (2002) 10, 666-668. doi:10.1038/sj.ejhg.5200857

Keywords: hypophosphatasia; founder effect; population genetics

\section{Introduction}

Hypophosphatasia (MIM nos. 146300, 241500 and 241510) is a rare inborn error of metabolism characterised by defective bone mineralisation and deficiency of serum and tissue liver/bone/kidney alkaline phosphatase (L/B/K ALP) activity. The disease is variable in its clinical expression, because of various mutations in the tissue-nonspecific alkaline phosphatase (TNSALP) gene. ${ }^{1}$ Severe forms of the disease (perinatal and infantile) are transmitted as an autosomal recessive trait while both autosomal recessive and autoso-

${ }^{*}$ Correspondence: E Mornet, Laboratoire SESEP, Batiment Fermat, Université de Versailles-Saint Quentin en Yvelines, 78035 Versailles Cedex, France. Tel: +3313925 46 76; Fax: +331 392546 78;

E-mail: etienne.mornet@cytogene.uvsq.fr

Received 7 February 2002; revised 24 May 2002; accepted 12 June 2002 mal dominant transmission may be found in milder forms. The TNSALP gene is localised on chromosome $1 \mathrm{p} 36.1-34^{2}$ and consists of 12 exons distributed over $50 \mathrm{~kb}^{3}$ More than 112 distinct mutations have been described in the TNSALP gene (see for review ${ }^{4}$ and the TNSALP gene mutations database at the internet address http://www.sesep.uvsq.fr/Database.html). Some of them were recurrently found in unrelated patients but only two may be considered as frequent: E174K $(571 \mathrm{G} \rightarrow \mathrm{A})$, which represents $7 \%$ of the tested Caucasian chromosomes, ${ }^{4}$ and 1159delT, which is frequently found in Japanese patients. ${ }^{5,6}$ The E174K mutation is mostly associated with mild (childhood, adult and odonto-) hypophosphatasia ${ }^{7,8}$ and was found in $31 \%$ of our patients affected with these clinical forms. Because the patients originated from various coun- 
tries, we investigated the likelihood of a unique origin or of multiple origins due to recurrence of de novo mutations. We genotyped three TNSALP gene intragenic polymorphisms, S93S $(330 \mathrm{C} \rightarrow \mathrm{T}), 472+12$ delG and V505A $(1565 \mathrm{C} \rightarrow \mathrm{T})$ in patients carrying $\mathrm{E} 174 \mathrm{~K}$ and in normal unrelated individuals, and compared haplotypic frequencies.

\section{Materials and methods}

Eighteen unrelated patients heterozygous for E174K and their relatives, and 30 unrelated normal individuals from the French population, were genotyped. We also genotyped 33 patients without the mutation $\mathrm{E} 174 \mathrm{~K}$ and their relatives. The patients live in various European countries and in North America and Australia. The E174K mutation in exon 6 of the TNSALP gene was detected by sequencing, according to a methodology previously reported. ${ }^{9}$ Three intragenic polymorphisms in exons 5 (S93S and $472+12$ delG) and 12 (V505A) were analysed by sequencing these two exons. The phases were determined by analysing the parents and/or the relatives of the patients. When the relatives did not allow determination of the phase at the E174K, S93S and 472+12delG loci, a PCR including exons 5 and 6 was performed by using the K174 allele-specific primer GGCTCAAGGCCTCAGGGGGCATCTT and the forward exon 5 primer CCTCACGCCCCAGTCCCCAT. These primers where annealed at $58^{\circ} \mathrm{C}$ for $40 \mathrm{~s}$ and extended for $4 \mathrm{~min}$ at $72^{\circ} \mathrm{C}$.

\section{Results}

Table 1 summarises the haplotypic frequencies found in normal and hypophosphatasia chromosomes. Among the eight expected possible haplotypes, only four were found in the general population: the major haplotypes C-G-T (71\%) and C-delG-T (17\%) from which derived the two rare haplotypes T-G-T (5\%) and C-G-C (7\%) (Table 1). A fifth haplotype T-delG-T (2\%) was found once in the group of hypophosphatasia chromosomes with mutation other than E174K. All the E174K mutations were associated with the TG-T haplotype, corresponding to alleles 330T, 472+12G and 1565T. This haplotype was rare in the control population (5\% of the tested chromosomes), clearly suggesting that the mutation occurred on a single ancestral chromosome carrying this haplotype and spread throughout various populations as a result of human migrations. The hypophosphatasia chromosomes without E174K showed allelic and haplotypic distributions similar to the controls $\left(X^{2}=2.33\right.$; $P \approx 0.53)$. The distributions of alleles and haplotypes were also similar in normal parental alleles $\left(X^{2}=1.83 ; P \approx 0.55\right)$, indicating that the use of unrelated French individuals as controls did not introduce any bias into the interpretation of the results. Indeed, these normal parental alleles completely matched hypophosphatasia chromosomes in their geographic distribution.

\section{Discussion}

The TNSALP gene is subject to strong allelic heterogeneity and most of the 112 mutations reported up to now were private. Mutation E174K is an exception, with a frequency of $7 \%$ in hypophosphatasia chromosomes and $31 \%$ in patients with mild hypophosphatasia, and raised the question as to whether the relatively high prevalence of this mutation was due to a genetic founder effect or to multiple mutational events. Our results show that the $18 \mathrm{E} 174 \mathrm{~K}$ mutations studied in our group occurred in the same rare haplotype, and that the E $174 \mathrm{~K}$ mutation is therefore the result of an ancestral mutation that occurred once. These results are consistent with the previous work of Henthorn et al. ${ }^{7}$ who reported another case of mild hypophosphatasia in a patient carrying $\mathrm{E} 174 \mathrm{~K}$ and the heterozygote marker allele 330T, which is strongly associated with haplotype A. It is therefore likely that this patient also carried the A haplotype.

Although the mutation seems to be more frequent in the north of Western Europe, it was also found, as other mild hypophosphatasia mutations, in various European countries, North America (USA and Canada) and Australia. This suggests that the mutation is relatively ancient and spread, through human migration, throughout Europe and was probably brought to the New World by European immigration. By comparison, the recurrent mutation A23V, associated with the common C-G-T haplotype, was found in seven patients with severe hypophosphatasia, six of them of German origin, and in the seventh patient was inherited from a North-American citizen originating from

Table 1 Haplotypic distribution of the three tested polymorphisms. Normal parental alleles correspond to normal chromosomes in parents of affected children carrying E174K. Other hypophosphatasia alleles correspond to chromosomes of patients with mutations other than E174K

\begin{tabular}{llllllll}
\hline Haplotype & $\begin{array}{l}\text { S93S } \\
(330 \mathrm{C} \rightarrow T)\end{array}$ & $472+12$ delG & $\begin{array}{l}\text { V505A } \\
(1565 T \rightarrow C)\end{array}$ & $\begin{array}{l}\text { Controls } \\
(\mathrm{n}=58)\end{array}$ & $\begin{array}{l}\text { E174K }(571 \mathrm{G} \rightarrow A) \\
\text { alleles }(\mathrm{n}=18)\end{array}$ & $\begin{array}{l}\text { Normal parental } \\
\text { alleles }(\mathrm{n}=20)\end{array}$ & $\begin{array}{l}\text { Other hypophosphatasia } \\
\text { alleles }(\mathrm{n}=66)\end{array}$ \\
\hline $\mathrm{A}$ & $\mathrm{T}$ & $\mathrm{G}$ & $\mathrm{T}$ & $0.05(n=3)$ & 1 & $0.10(n=2)$ & $0.06(n=4)$ \\
B & $\mathrm{T}$ & delG & $\mathrm{T}$ & 0 & 0 & $0.02(n=1)$ \\
C & $\mathrm{C}$ & $\mathrm{G}$ & $\mathrm{T}$ & $0.71(n=41)$ & 0 & $0.65(n=13)$ & $0.70(n=46)$ \\
$\mathrm{D}$ & $\mathrm{C}$ & $\mathrm{G}$ & $\mathrm{C}$ & $0.07(n=4)$ & 0 & 0 & $0.09(n=6)$ \\
E & $\mathrm{C}$ & delG & $\mathrm{T}$ & $0.17(n=10)$ & 0 & $0.25(n=5)$ & $0.13(n=9)$ \\
\hline
\end{tabular}

$n$ : number of chromosomes studied. 
Poland and Czechoslovakia. By contrast with the more frequent E174K mutation, $\mathrm{A} 23 \mathrm{~V}$ is therefore confined to a small geographic region, suggesting that it resulted from a more recent ancestral mutational event and that the mutation has not yet spread through other European countries.

\section{References}

1 Whyte MP: Hypophosphatasia and the role of alkaline phosphatase in skeletal mineralization. Endocr Rev 1994; 15: 439-461.

2 Greenberg CR, Evans JA, McKendry-Smith S et al: Infantile hypophosphatasia localization within chromosome region 1p36.1.134 and prenatal diagnosis using linked DNA markers. Am J Hum Genet 1990; 46: 286-292.

3 Weiss MJ, Ray K, Henthorn PS, Lamb B, Kadesch T, Harris H: Structure of the human liver/bone/kidney alkaline phosphatase gene. J Biol Chem 1988; 263: $12002-12010$.

4 Mornet E: Hypophosphatasia: the mutations of the tissuenonspecific alkaline phosphatase gene. Hum Mutat 2000; 15: $309-315$.
5 Goseki-Sone M, Orimo H, Iimura T et al: Hypophosphatasia: identification of five novel mutations (G507A, G705A, A748G, T1155C, G1320A) in the tissues-nonspecific alkaline phosphatase gene among Japanese patients. Hum Mut 1998; 1(Suppl): S263S267.

6 Orimo $\mathrm{H}$, Goseki-Sone $\mathrm{M}$, Inoue $\mathrm{M}$, Tsubakio $\mathrm{Y}$, Sakiyama T, Shimada T: Importance of deletion of $\mathrm{T}$ at nucleotide 1559 in the tissue-nonspecific alkaline phosphatase gene in Japanese patients with hypophosphatasia. I Bone Miner Metab 2002; 20 $28-33$.

7 Henthorn PS, Raducha M, Fedde KN, Lafferty MA, Whyte MP: Different mutations at the tissue-nonspecific alkaline phosphatase gene locus in autosomal recessively inherited forms of mild and severe hypophosphatasia. Proc Natl Acad Sci USA 1992; 89 9924-9928.

8 Zurutuza L, Muller F, Gibrat JF et al: Correlations of genotype and phenotype in hypophosphatasia. Hum Mol Genet 1999; 8: 10391046.

9 Mornet E, Taillandier A, Peyramaure S et al: Identification of fifteen novel mutations in the tissue-nonspecific alkaline phosphatase (TNSALP) gene in European patients with severe hypophosphatasia. Eur J Hum Genet 1998; 6: 308-314. 